\title{
Establishment of hepatic and neural differentiation platforms of Wilson's disease specific induced pluripotent stem cells
}

\author{
Fei Yi ${ }^{1}$, Jing $Q u^{1}$, Mo Li ${ }^{1}$, Keiichiro Suzuki ${ }^{1}$, Na Young Kim ${ }^{1}$, Guang-Hui Liu ${ }^{1,2}$, Juan Carlos Izpisua Belmonte ${ }^{1,3 凶}$ \\ ${ }^{1}$ Gene Expression Laboratory, Salk Institute for Biological Studies, 10010 North Torrey Pines Road, La Jolla, CA 92037, USA \\ ${ }^{2}$ National Laboratory of Biomacromolecules, Institute of Biophysics, Chinese Academy of Sciences, Beijing 100101, China \\ ${ }^{3}$ Center for Regenerative Medicine in Barcelona, Dr. Aiguader 88, 08003 Barcelona, Spain \\ $\triangle$ Correspondence: belmonte@salk.edu or izpisua@cmrb.eu \\ Received July 2, 2012 Accepted July 13, 2012
}

\begin{abstract}
The combination of disease-specific human induced pluripotent stem cells (iPSC) and directed cell differentiation offers an ideal platform for modeling and studying many inherited human diseases. Wilson's disease (WD) is a monogenic disorder of toxic copper accumulation caused by pathologic mutations of the ATP7B gene. WD affects multiple organs with primary manifestations in the liver and central nervous system (CNS). In order to better investigate the cellular pathogenesis of WD and to develop novel therapies against various WD syndromes, we sought to establish a comprehensive platform to differentiate WD patient iPSC into both hepatic and neural lineages. Here we report the generation of patient iPSC bearing a Caucasian population hotspot mutation of ATP7B. Combining with directed cell differentiation strategies, we successfully differentiated WD iPSC into hepatocyte-like cells, neural stem cells and neurons. Gene expression analysis and cDNA sequencing confirmed the expression of the mutant ATP7B gene in all differentiated cells. Hence we established a platform for studying both hepatic and neural abnormalities of WD, which may provide a new tool for tissue-specific disease modeling and drug screening in the future.
\end{abstract}

KEYWORDS induced pluripotent stem cell, Wilson's disease, hepatocyte, neural stem cell, neuron

\section{INTRODUCTION}

Wilson's disease (WD) is an autosomal recessive disorder of copper metabolism caused by monogenic mutations. The disease affects between 1 in 30,000 and 1 in 100,000 individuals, and invariably results in severe disability and death if untreated (Ala et al., 2007). The disease gene ATP7B encodes for a copper transporting P-type ATPase which is critical in the distribution and elimination of excess copper from cells. Various WD associated mutations of ATP7B disrupt the transport activity of the protein, which also results in protein mislocalization and reduced stability (Huster et al., 2012). WD is characterized by the toxic accumulation of copper in the liver and subsequently in the central nervous system (CNS) and other organs, thereby causing severe liver damage and/or neurological abnormality. Excessive copper can also accumulate in other parts of the body including eyes and kidneys. To date, molecular genetics analysis has revealed more than 500 different mutations of ATP7B associated with WD (http://www.wilsondisease.med.ualberta.ca/database.asp) (Kenney and Cox, 2007).

The liver manifestation of WD often presents in childhood into early adulthood. Hepatic copper homeostasis is dependent on both copper transport activity and localization. ATP7B is in charge of delivering copper to apo-ceruloplasmin within the trans-Golgi network, and transporting excessive copper out of hepatocytes for the subsequent excretion via the bile (Murata et al., 1995; Terada et al., 1999; Forbes and Cox, 2000). The main biochemical defect of WD in liver is the hepatic copper accumulation caused by the disrupted biliary copper efflux (Forbes and Cox, 2000). Numerous diseaserelated mutations of ATP7B have been identified and suggested to correlate with the hepatic manifestations of WD. However, the hepatic copper levels of WD patients may vary 
among individuals depending on bioavailability and genetic factors. The mechanisms behind this variability are complex. Robust genotype-phenotype correlation of missense mutation of ATP7B in WD has not been clearly characterized yet (Schilsky, 2009).

In addition to the more common manifestation in the liver, WD also affects the CNS at high prevalence. When first discovered in 1912, WD was described by a neurologist as a familial nervous disease causing progressive lenticular degeneration (Wilson, 1912). Previous research has indicated that $40 \%-50 \%$ of WD patients will develop pathological symptoms in the CNS (Walshe, 1962). Neurological signs of WD generally tend to be developed later than the hepatic symptoms. Patients with the CNS affected are often believed to have liver disease at the time of presentation, but they may not be symptomatic from the liver disease (Ala et al., 2007). It has been proposed that some genotype of WD, like the most common mutation in the Caucasian population $\mathrm{H} 1069 \mathrm{Q}$, is associated with a later onset of neurological disease; however, the correlation of neurological manifestation with most genotypes of WD is still elusive (Stapelbroek et al., 2004; Lorincz, 2010).

The fact that WD presents with various phenotypes and affects different organs complicates the diagnostic process and treatment. Once the diagnosis of WD is established, except for the option of orthotopic liver transplantation, a lifelong treatment with copper chelator or zinc salts is necessary. Furthermore, the mechanisms underlying its hepatic and neural manifestations are still largely elusive, which jeopardizes the discovery of new drug or novel therapy for WD. The growing knowledge of the disease gene ATP7B and the advancing of the human induced pluripotent stem cell (iPSC) technology have opened up new opportunities to model and study WD. The strategy to generate iPSC from WD patients with disease-specific mutations and the subsequent differentiation of patient iPSC into various disease-affected cell types renders a promising platform in vitro to better investigate the pathogenesis of WD in different tissues as well as to potential gene correction and cell-based therapy in the future. Recently, the generation of human iPSC harboring an Asian hotspot mutation of WD has been reported (Zhang et al., 2011). Here we report the generation of human iPSC from a novel Caucasian hotspot mutation of WD and the establishment of hepatic and neural platforms using the WD iPSC.

\section{RESULTS}

WD patient primary fibroblasts were obtained from Telethon Genetic Biobank Network (http://dppm.gaslini.org/biobank/, Italy). Patient fibroblasts were expanded in vitro and the genotypes were examined by genomic DNA extraction and sequencing. An ATP7B M769V homozygous mutant patient fibroblast line (FFF0101990) was chosen as an example for further studies due to its high prevalence in Europe. The
M769V mutation (ATG to GTG) is a disease-associated missense mutation of ATP7B gene (Kenney and Cox, 2007). It is located in the frequently mutated exon 8 and is one of the hotspot mutations of WD in the Caucasian population (Curtis et al., 1999; Huster et al., 2004; Lepori et al., 2007). Biochemical analysis has revealed a significantly impaired transport activity of the M769V mutant ATP7B (Huster et al., 2012). We firstly reprogrammed the patient fibroblasts to iPSC by retroviruses encoding Oct4, Sox2, KIf4, c-Myc and green fluorescent protein (GFP) (Takahashi et al., 2007). iPSC-like colonies emerged three weeks after transduction coinciding with the loss of GFP expression, which is an indicator of epigenetic silencing of retroviral-transgenes in pluripotent cells (Fig. 1A). iPSC colonies were manually picked and expanded in feeder-free condition on matrigel. The WD patient-origin of the established iPSC lines were attested by genotyping specifically to the M769V point mutation (Fig. 1B). WD iPSC exhibit typical human pluripotent stem cell morphology and ubiquitously expressed pluripotent markers Oct4 Sox2 and the surface antigen TRA-1-60 (Figs. 1A and 1C). The pluripotency of the WD iPSC was examined by embryoid body (EB) based spontaneous differentiation in vitro, and differentiation into all three primary lineages was detected (Fig. 1D).

Among all WD affected organs, liver and brain are the ones with the highest prevalence. We firstly sought to differentiate WD iPSC into the hepatic lineage. We utilized a previously established protocol with a few improvements to differentiate WD iPSC into hepatocyte-like cells (HLC) through three hepatic developmental stages (Song et al., 2009). WD iPSC exhibited morphological changes through the proceeding of differentiation and the hepatic progenitor lineage commitment was demonstrated by the classical cubical cell shape (Fig. 2A). Immunofluorescent staining showed the homogenous expression of hepatoblast markers FoxA2 and $\mathrm{Hnf} 4 \alpha$ at day 15 following differentiation (Fig. 2B). In order to better characterize the differentiation and maturation status of the HLC generated, we performed quantitative PCR (qPCR) to elucidate the gene expression profiles. Pluripotent markers Nanog and Oct4 were completely silenced after differentiation, and WD iPSC differentiated HLC showed dramatic induction of hepatic markers comparing to primary human hepatocytes $(\mathrm{PHH})$ at D18 and D25 of differentiation (Fig. 2C). The overexpression of alpha-fetoprotein (AFP) implied a fetal-stage immature character of the differentiated HLC, while a less but significant level of hepatocyte functional protein albumin (ALB) was also observed. Moreover, the WD HLC started to express considerable levels of markers specific for mature hepatocytes, the liver-specific dioxygenase Tdo2 and the most important cytochrome P450 enzyme CYP3A4, which indicated their relative maturation (Fig. 2C). We next sought to perform in vitro functional assay to further attest the functionality of the differentiated WD HLC. Periodic Acid Schiff (PAS) staining clearly demonstrated the glycogen storage 
A Emerging of iPSC colony Silencing of transgene (GFP)

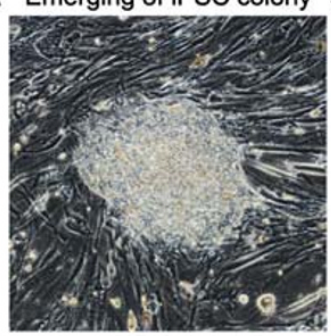

iPSC clone \#1

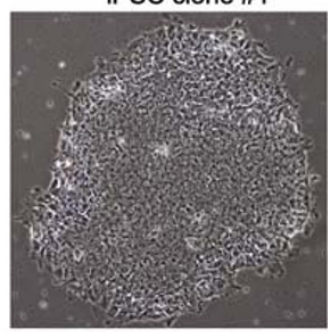

iPSC clone \#2

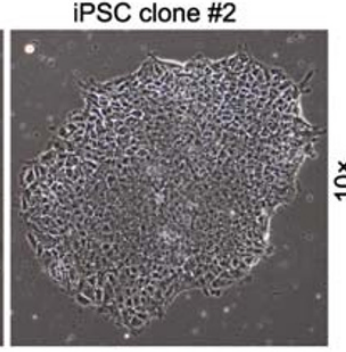

C

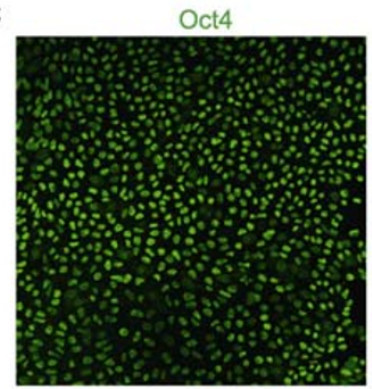

TRA-1-60

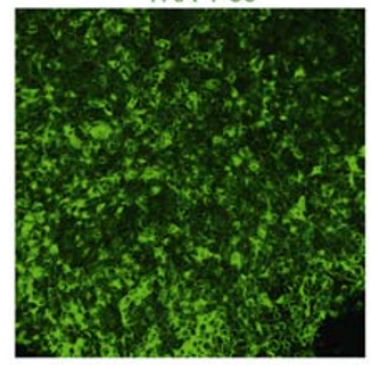

Sox2

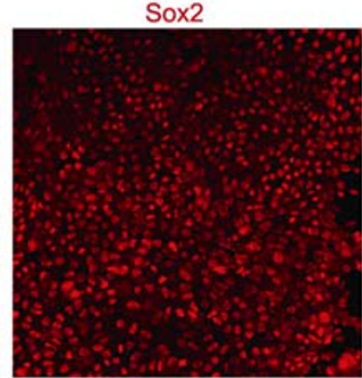

Oct4

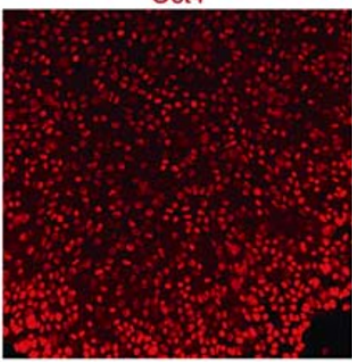

ATP7B-WT Fib
C C C C AT G C T $\operatorname{CCCAT}$

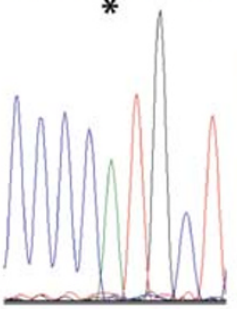

ATP7B-M769V Fib ATP7B-M769V iPSC C C C CG T G C T C C CG TG CT
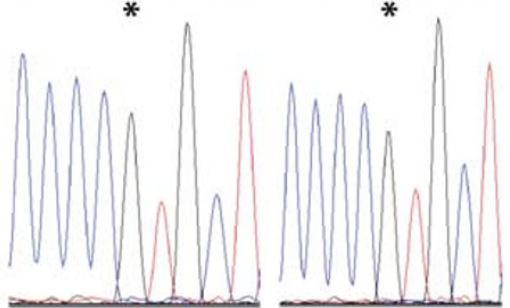

$\stackrel{x}{\circ}$

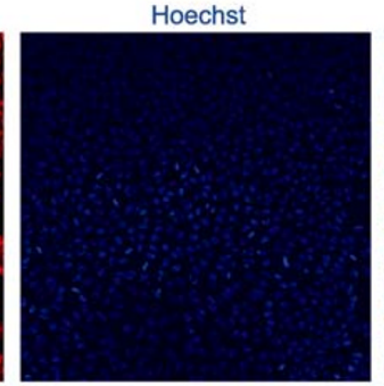

Hoechst

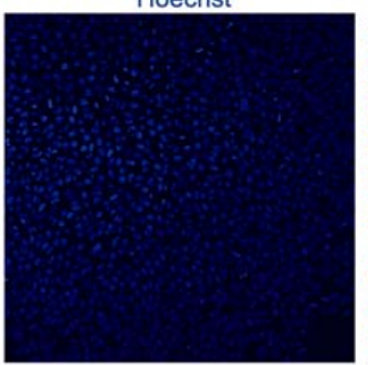

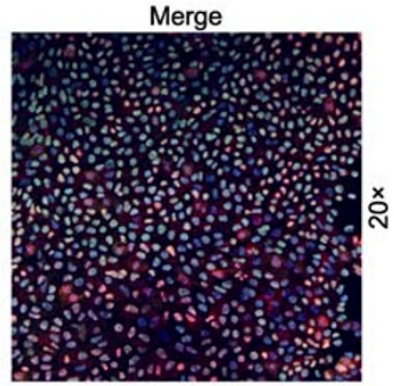

Merge

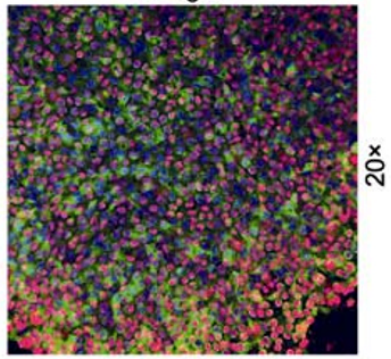

D

Ectoderm: Tuj1/Hoechst

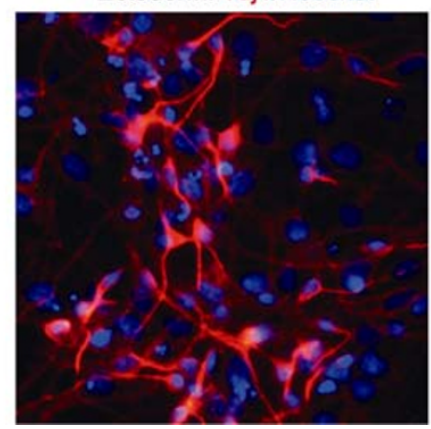

Mesoderm: SMA/Hoechst

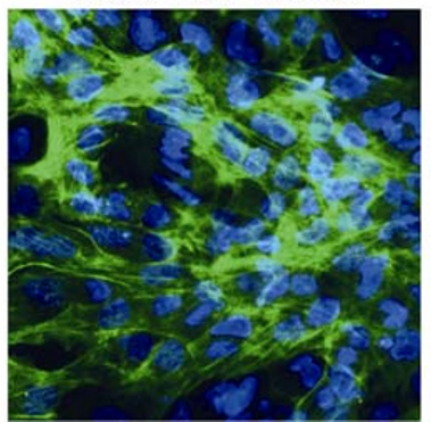

Endoderm: FoxA2/Hoechst

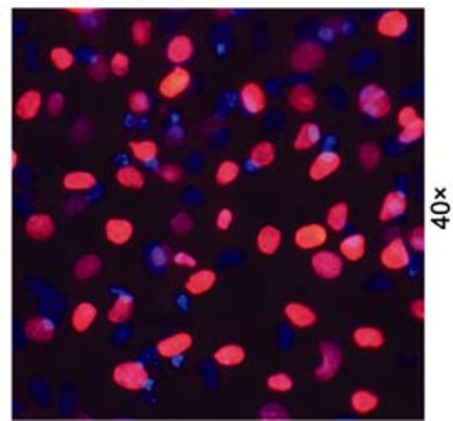

Figure 1. Generation of human WD-specific iPSC. (A) Generation of human iPSC from WD patient's fibroblasts. Loss of retro viral-GFP expression indicates the silencing of reprogramming retroviruses. Morphologies of two typical clones of established iPSC lines are shown. (B) Genotyping results showing the homozygous A to G mutation in ATP7B M769V mutant iPSC. (C) Immunofluorescence staining for pluripotent markers in WD iPSC. Nuclei are stained in blue with Hoechst (also thereafter). (D) Immunofluorescence staining showing the EB-based spontaneous differentiation of WD iPSC. Markers of all three primary germ layers were detected. 
A

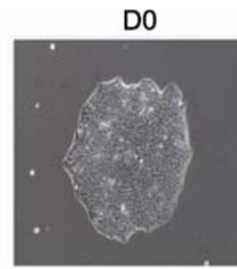

D7
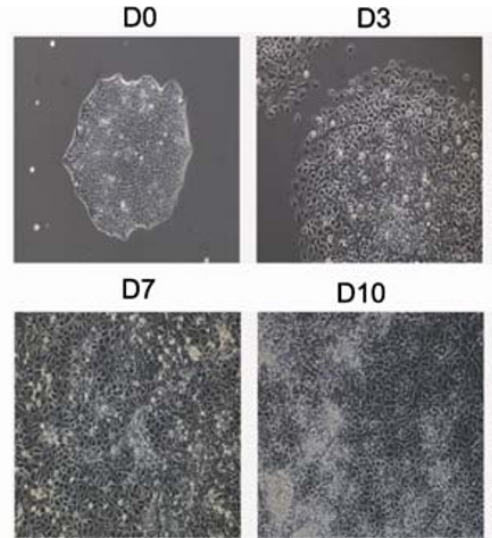

D10
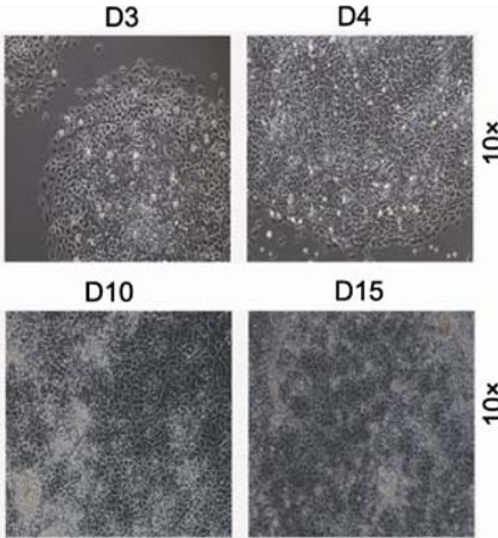

D15

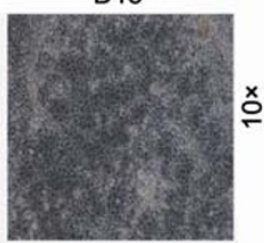

C
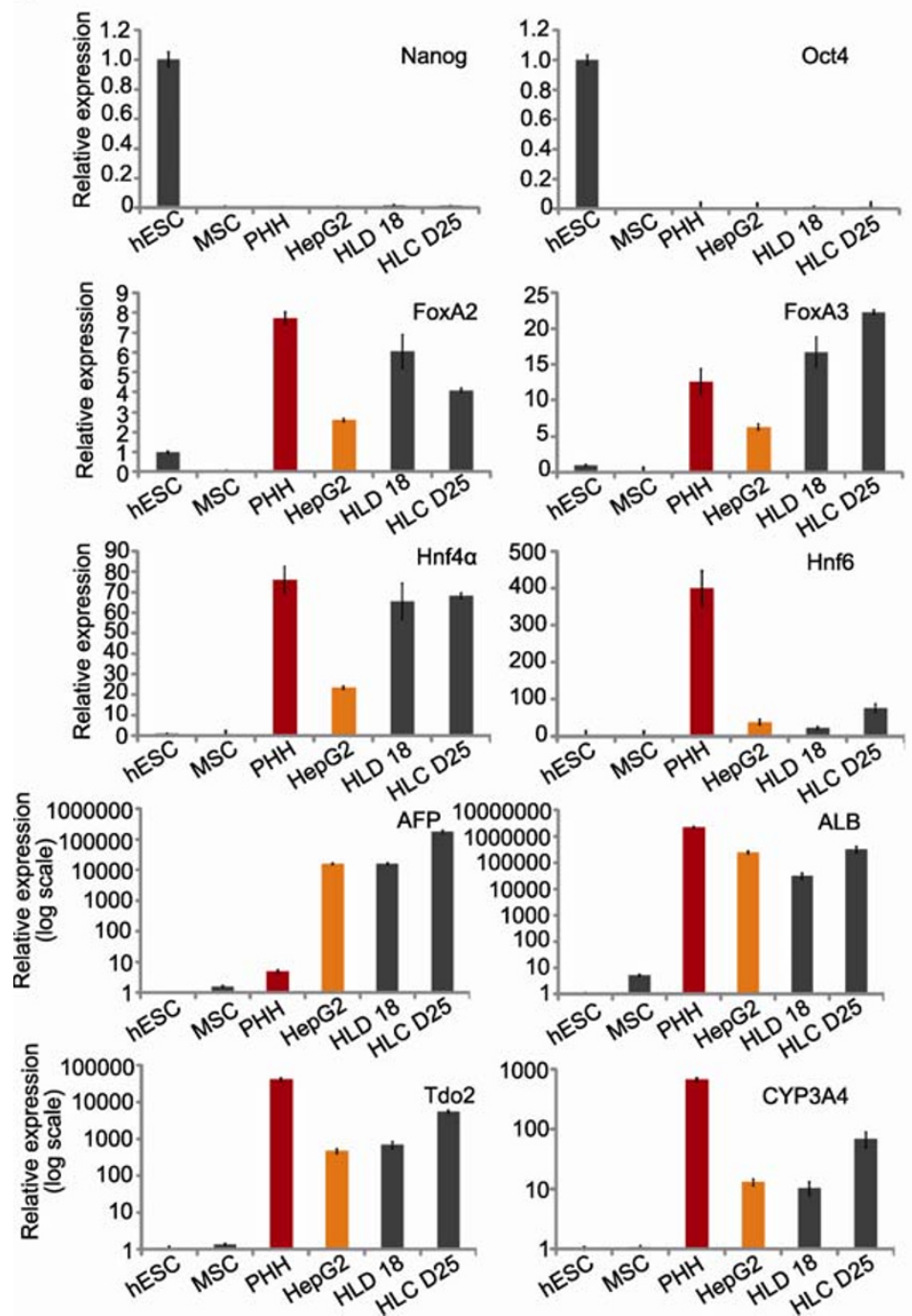

\section{0}
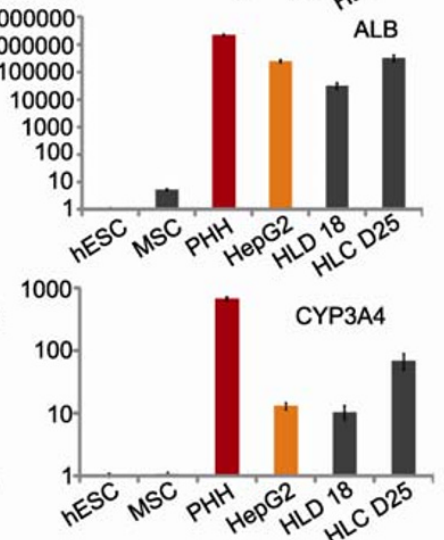
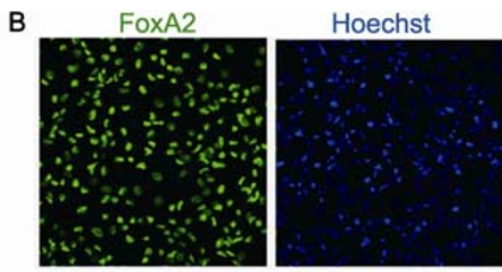

Hoechst
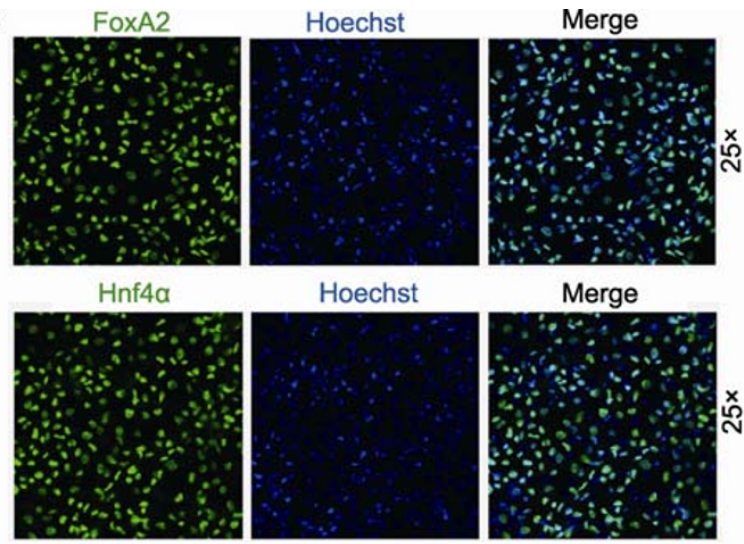

Merge

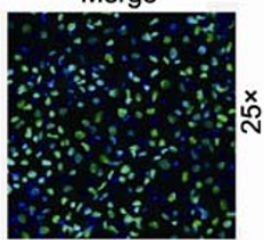

D

PAS staining
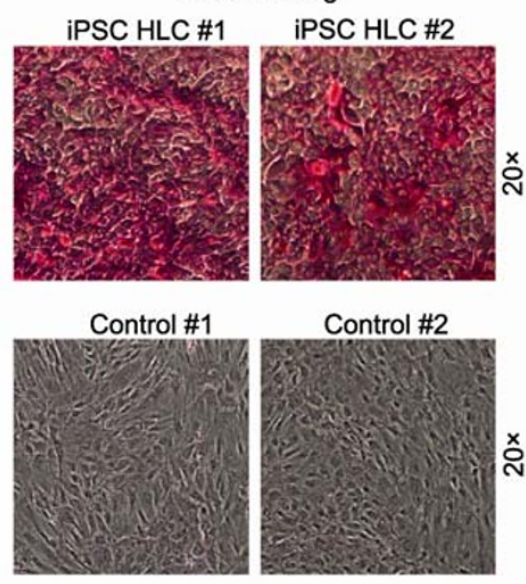

E

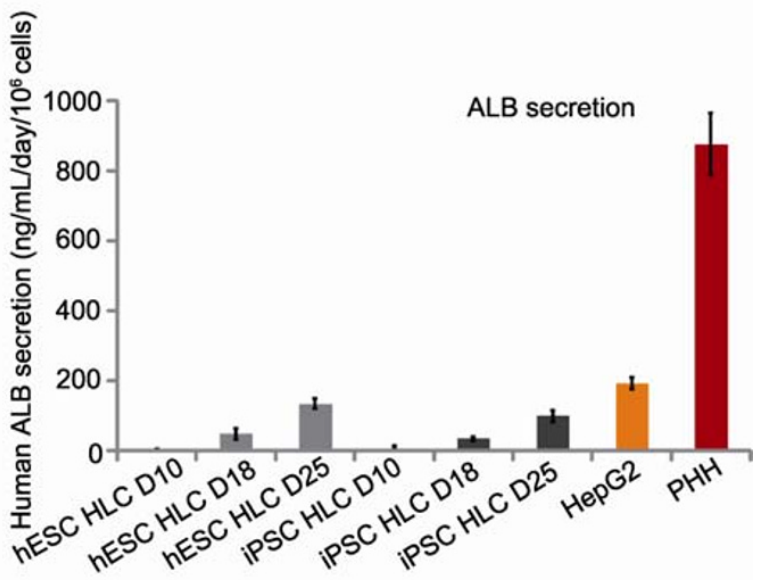

Figure 2. Differentiation of iPSC into WD-specific HLC. (A) Bright-field microscopy showing the stepwise morphology changes of HLC differentiation from WD iPSC. (B) Immunofluorescence staining showing the homogenous expression of hepatoblast markers FoxA2 and Hnf4a at day 15 of differentiation. (C) qPCR analysis for expressions of selected pluripotent and hepatic genes in HLC differentiation at indicated time points. Relative expressions (mean of three replicates) were normalized to levels in hESC. PHH cDNA was used as the positive control of full hepatic maturation. (D) PAS staining for the glycogen storage in WD iPSC derived HLC at day 25. (E) ELISA analysis for ALB secretion in WD iPSC derived HLC at day 10, 18 and 25. 
ability of WD HLC (Fig. 2D). Furthermore, ELISA-based ALB secretion confirmed the significant levels of ALB production in WD HLC and the comparable hepatic differentiation efficiency to the human embryonic stem cells (hESC) derived HLC (Fig. 2E). Therefore, we demonstrated that HLC can be efficiently differentiated from WD iPSC and are functional in vitro.

Besides the liver, the CNS is the second most commonly affected organ by WD. In order to better facilitate the neurological-specific pathological studies of WD, we next sought to differentiate WD iPSC into neural lineages. We firstly differentiated the WD iPSC into self-renewable neural stem cells (NSC) based on a recently reported method under chemically defined condition (Li et al., 2011b). The WD iPSC derived NSC were homogenous in neuroepithelial morphology and expandable with a high repopulation kinetics (Fig. $3 A$ ). Similarly to their counterparts from hESC, WD iPSC derived NSC homogenously expressed high levels of NSC markers Nestin and Pax6 (Fig. 3B). Importantly, since the neurological abnormalities of WD often manifest with aging, the establishment of self-renewable WD NSC renders a new opportunity for modeling aging-associated neurological abnormalities of WD in vitro. In addition, the neuron differentiation potential of the NSC was examined by pan-neural differentiation. Within 7 days, the passage 4 WD iPSC-derived NSC exhibited high efficiency of differentiating into pan-neurons, closely resembling their counterparts differentiated from hESC (Fig. 3C). More interestingly, differentiation into Tyrosine hydroxylase (TH)-positive dopaminergic neu- rons was also observed (Fig. 3D). It has been previously suggested that neurological WD correlates with deficit of dopaminergic neurons and may contribute to midbrain atrophy (Barthel et al., 2003; Strecker et al., 2006). Therefore, the generation of WD specific dopaminergic neurons may provide an ideal platform for better investigation of the midbrain specific neurological defects of WD in vitro.

We next investigated if the mutant ATP7B is expressed in the differentiated cell types. The qPCR data revealed that the expression of ATP7B was highly induced in differentiated HLC, NSC and pan-neurons, compared to the undifferentiated status (Fig. 4A). At the end, we designed primers to specifically amplify the mutation containing region from cDNA obtained from each cell type. The expressions of M769V mutant ATP7B cDNA transcripts were confirmed through CDNA sequencing (Fig. 4B). These data suggest the mutant ATP7B is highly expressed in differentiated hepatic and neural cells, which endows both platforms great value for modeling and studying the hepatic and neural symptoms of WD in vitro.

\section{DISCUSSION}

Since first established in 2007, human iPSC have been widely used for modeling human genetic disorders. Combining with the recent advances in gene targeting technology, we have previously showed that human iPSC can recapitulate pathological defects and be genetically corrected from
A

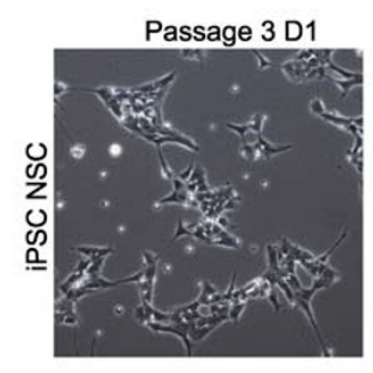

C

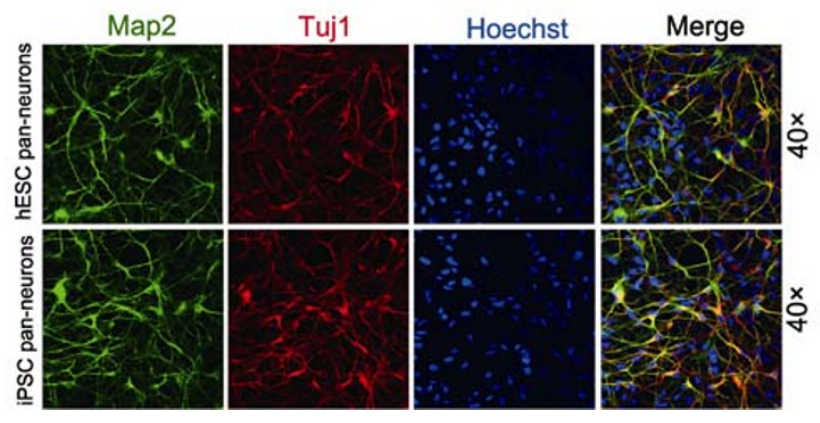

Passage 3 D3

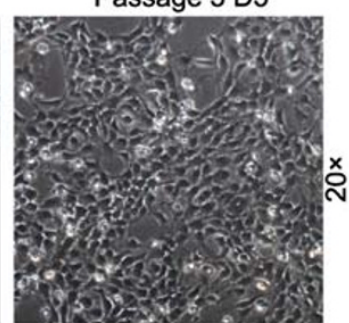

B

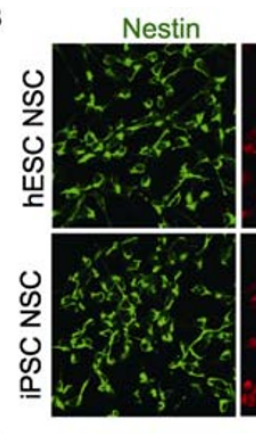

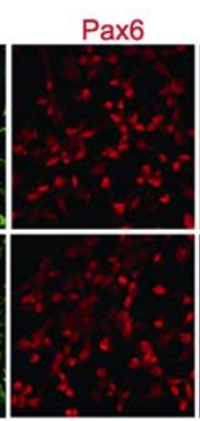
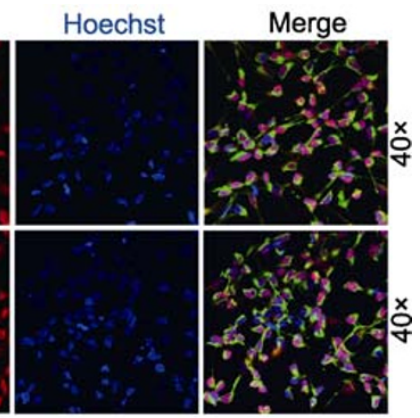

D
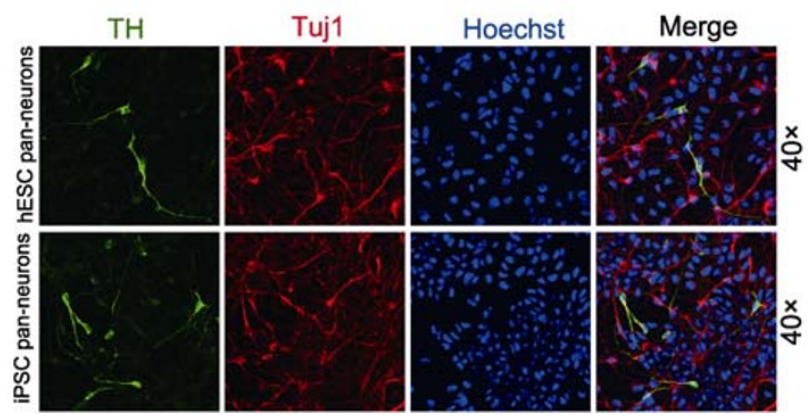

Figure 3. Differentiation of iPSC into WD-specific NSC and neurons. (A) Bright-field microscopy showing the morphology and proliferation of WD iPSC derived NSC at passage 3. (B) Immunofluorescence staining showing the homogenous expression of neural progenitor markers Nestin and Pax6. (C) Immunofluorescence staining showing the pan-neuron differentiation from WD iPSC derived NSC.

(D) Detection of TH-positive dopaminergic neurons in pan-neuron differentiation of NSC. 
A

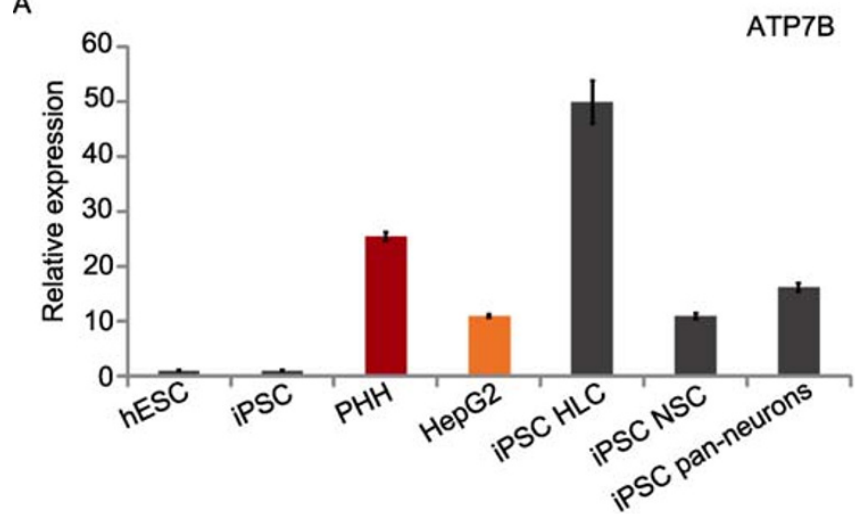

C

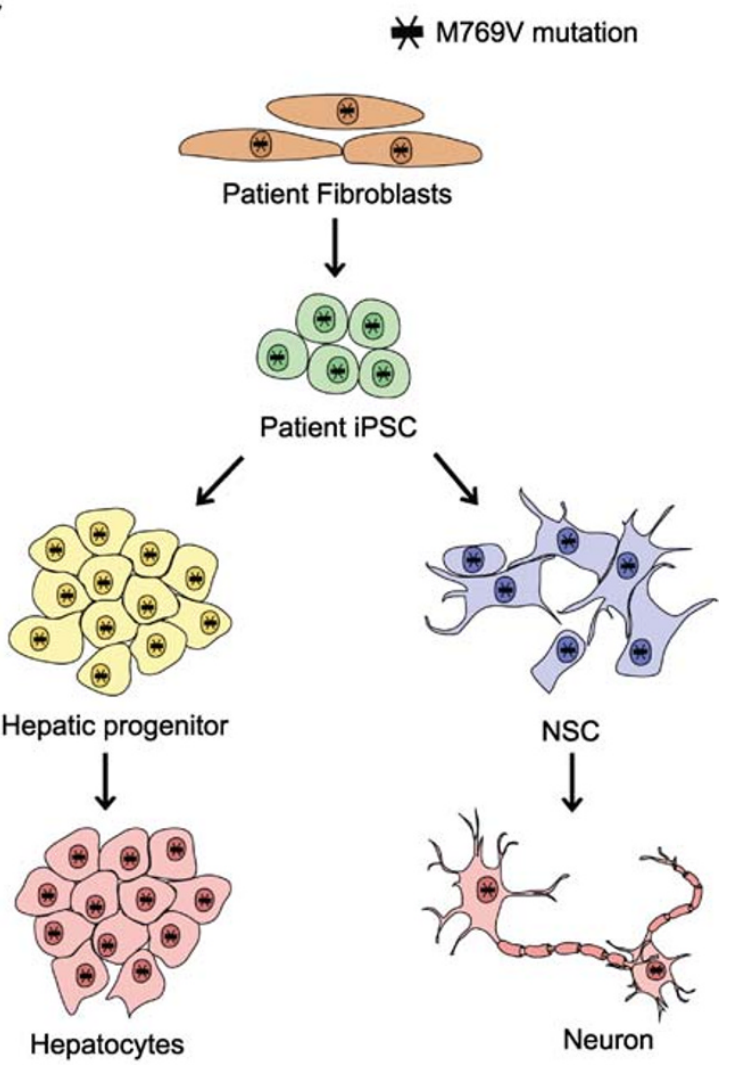

Figure 4. Detection of mutant ATP7B expression in hepatic and neural lineages. (A) Detection of ATP7B expression in hepatic and neural cells derived from WD iPSC. Relative expressions (mean of three replicates) were normalized to the level in $\mathrm{hESC}$. (B) Detection of homozygous ATP7B M769V mutant expression in hepatic and neural cells derived from WD iPSC. (C) Scheme demonstrating the strategy of establishing WD specific mutation containing hepatic and neural platforms from WD iPSC.

patients suffering monogenic diseases (Li et al., 2011a; Liu et al., 2011a, 2011b). Similarly to many other reports of iPSC-based disease modeling, efficient differentiation of patient-specific iPSC into disease-affected cell types represents a critical challenge to the success of the study. Among many established differentiation protocols, efficient differentiation of human pluripotent stem cells into functional hepatocytes is technically challenging, in spite of its tremendous application potential (Yi et al., 2012). In line with many other reports, we observed that the HLC differentiated from patient iPSC resemble many important features to $\mathrm{PHH}$ and exhibit functionality in vitro, however, they do not fully match the maturation level of $\mathrm{PHH}$. On the other hand, the human iPSC-derived HLC do have therapeutic potential, as it has been shown that human iPSC-HLC have functional and proliferative potentials in vivo after transplantation into an acute liver damage animal model (Chen et al., 2012).

In many human genetic diseases, the inherited disorders often affect multiple cell types in different organs. Therefore, the successful establishment of different lineage-committed platforms is not only important but also required for a comprehensive investigation of the disease. Besides hepatic symptoms, manifestations in the CNS count second in all WD cases. Although the modeling of hepatic defects of WD using iPSC has been previously demonstrated (Zhang et al., 2011), to our best knowledge, the establishment of neural lineages harboring ATP7B pathological mutation has never been reported. It has long been suggested that ATP7B might play a key roles in neurotransmissions. In addition, the neurotoxicity caused by abnormal copper accumulation in the critical brain regions by mutation of the ATP7B gene may lead to neurological defects of WD (Saito et al., 1999). However, the pathological correlation of genotype-phenotype for neurologic WD is still largely unknown. Therefore, the generation of expandable WD specific NSC and neurons may open up new opportunities to better understand the pathogenesis of neurologic WD. Furthermore, as the WD often causes Tremor, Choreoathetosis and Parkinsonism-like syndromes in patients, the differentiation of various types of neurons including TH-positive dopaminergic neurons harboring WD specific mutation may potentially be useful for investigating the cellular mechanism of the WD associated neurologic syndromes.

As shown in this study, we report the successful generation of iPSC with a novel human ATP7B hotspot mutation and 
the establishment of both hepatic and neural differentiation platforms for WD-specific iPSC (Fig. 4C). We observed that the mutant ATP7B gene is highly expressed in differentiated cells, which endows the value and potential application of using these cells for future studies, including disease modeling, drug screening and gene correction to generate disease-free cells for potential cell replacement therapy. In addition, the methodology and platforms established in our study can be readily applied to other hotspot mutations (e.g. H1069Q, R778L, et al.) of WD. Taken together, our study may provide new tools to facilitate the modeling of $W D$ in vitro and may be well-suited for further follow-up studies in diverse fields of WD research.

\section{MATERIALS AND METHODS}

\section{Cell culture}

The human fibroblasts were cultured in DMEM (Invitrogen) containing $15 \%$ fetal bovine serum (FBS, Gemini), $0.1 \mathrm{mmol} / \mathrm{L}$ non-essential amino acids (Invitrogen), $1 \mathrm{mmol} / \mathrm{L}$ glutamax (Invitrogen), and 1 $\mathrm{mmol} / \mathrm{L}$ sodium pyruvate (Invitrogen). H9 hESCs (WiCell Research) and IPSCs were maintained on a layer of mitotically inactivated mouse embryonic fibroblasts (MEFs) in hESC medium:

DMEM/F12 (Invitrogen) supplemented with $0.1 \mathrm{mmol} / \mathrm{L}$ non-essential amino acids (Invitrogen), $1 \mathrm{mmol} / \mathrm{L}$ GlutaMAX (Invitrogen), 20\% Knockout Serum Replacement (Invitrogen), $55 \mu \mathrm{mol} / \mathrm{L}$ $\beta$-mercaptoethanol (Invitrogen) and $10 \mathrm{ng} / \mathrm{mL} 21 \mathrm{bFGF}$ (Joint Protein Central). hESCs and iPSCs were also cultured in Matrigel (BD Biosciences) with mTeSR medium (StemCell Technologies).

\section{Generation of iPSC}

For retrovirus production, 293T cells were transfected with the pMXs vectors carrying OCT4, SOX2, c-MYC, KLF4 or GFP CDNAs, together with the packaging plasmids (pCMV-gag-pol-PA and pCMV-VSVg) using Lipofectamine 2000 (Invitrogen). Retroviruses were collected $36-48 \mathrm{~h}$ after transfection, and filtered through a $0.45-\mu \mathrm{mol} / \mathrm{L}$ filter. For the generation of human iPSCs, human fibroblasts were seeded in a 6-well plate and spin-infected with a mix of high-quality retroviruses encoding OCT4, SOX2, KLF4, C-MYC and GFP in the presence of $4 \mu \mathrm{g} / \mathrm{mL}$ polybrene. Three infections on consecutive days were performed. Six days after the first infection, fibroblasts were gently individualized with TrypLE (Invitrogen) and seeded onto fresh MEFs in the fibroblast culture medium. After $24 \mathrm{~h}$, the medium was switched to hESC medium, and changed every 1-2 days depending on cell density. To establish the iPSC lines, colonies were manually picked and transferred onto MEFs feeder cells for several passages before being transferred to Matrigel/mTeSR conditions.

\section{EB based iPSC differentiation}

For embryoid bodies (EBs) based differentiation, the iPSC colonies growing on MEFs were detached with dispase treatment, resuspended in DMEM/F12 medium supplemented with $0.1 \mathrm{mmol} / \mathrm{L}$ non-essential amino acids, $0.5 \mathrm{mmol} / \mathrm{L}$ L-glutamine, 10\% FBS (Atlanta Biologicals), and $50 \mu \mathrm{mol} / \mathrm{L} \beta$-mercaptoethanol and cultured in low attachment 6 -well plates for 4 days. The EBs were then plated on gelatin-coated plates and maintained for another 7-10 days.

\section{Immunofluorescence staining}

$4 \%$ formaldehyde in PBS was used to fix cells at room temperature (RT) for 20-30 min. After fixation, cells were exposed to $0.4 \%$ Triton X-100 in PBS for 5 min at RT. Cells were blocked with 10\% FBS in PBS for 30 min and incubated with primary antibody for $1 \mathrm{~h}$ at RT or overnight at $4^{\circ} \mathrm{C}$. Washing was conducted with PBS followed by incubation with a corresponding secondary antibody (Invitrogen) for 1 $h$ at RT. Hoechst 33342 was used to stain nuclei. Primary antibodies were obtained from the following sources. Santa Cruz Biotechnology: anti-Oct4 (sc-5279 and sc-8628), anti-Sox2 (sc-17320); anti-TRA-1-60 (sc-21705); Cell Signaling Technology: anti-FoxA2 anti-AFP (\#3143), anti-Hnf4a (\#3113); Sigma: anti-Tuj1 (T2200), anti-SMA (A5228), anti-Map2 (M4403); Abcam: anti-Pax6 (ab5790); Pel-Freez: anti-Tyrosine Hydroxylase (P40101); Millipore: anti-Nestin (MAB5326); Covance: anti-Tuj1 (MMS-435P).

\section{mRNA analysis and quantitative PCR}

Total RNA was extracted using TRIzol (Invitrogen) followed by cDNA synthesis using iScript reverse transcription supermix (Bio-Rad). Quantitative PCR were performed using SsoAdvanced SYBR Green Supermix on CFX384 Touch Real-Time PCR detection system (Bio-Rad). Primer sequences are listed in Table S1.

\section{Hepatocyte-like cells differentiation}

Differentiation of iPSC towards HLC was based on a previous report with minor modifications (Song et al., 2009). The differentiation was started on small colonies of iPSC cultured on Matrigel. For the first 3 days of definitive endoderm induction, $50 \mathrm{ng} / \mathrm{mL}$ Wn3a was added together with $100 \mathrm{ng} / \mathrm{mL}$ Activin A. During the hepatic induction, 20 $\mathrm{ng} / \mathrm{mL}$ BMP4 was used instead of $20 \mathrm{ng} / \mathrm{mL}$ BMP2. All cytokines were purchased from Peprotech. PAS staining of HLC was performed using a kit purchased from Sigma. ALB secretion was performed using Human Albumin ELISA Quantitation Set from Bethyl Laboratory. Primary human hepatocytes were obtained from Celsis In Vitro Technologies.

\section{Neural stem cells and neuron differentiation}

The generation and passaging of self-renewable NSC from iPSC was based on a previous report ( $\mathrm{Li}$ et al., 2011b). For the spontaneous neuronal differentiation, $2000 \mathrm{NSCs}$ were plated onto a Matrigel-coated $35 \mathrm{~mm}$ well, and maintained in NSC maintenance medium for 3 days. The NSC were then switched into differentiation medium containing DMEM/F12, $1 \times$ N2, 1× B27 (Invitrogen), 400 $\mu \mathrm{mol} / \mathrm{L}$ dcAMP (Sigma), $200 \mu \mathrm{mol} / \mathrm{L}$ Ascorbic acid (Sigma), $10 \mathrm{ng} / \mathrm{mL}$ BDNF (Peprotech), and $10 \mathrm{ng} / \mathrm{mL}$ GDNF (Peprotech). Two days after being switched to differentiation medium, laminin (Sigma) was added to the cells to promote differentiation. Cells were maintained in differentiation medium for 14 days in total, and then immunostained for 
neuronal markers.

\section{ACKNOWLEDGEMENTS}

We thank our funding agencies who supported this study. Work in the laboratory of JCIB was supported by Sanofi-Aventis, The Helmsley Charitable Trust and The Ellison Medical Foundation. G.H.L. was supported by "Thousand Young Talents" program of China, National Laboratory of Biomacromolecules, Strategic Priority Research Program of the Chinese Academy of Sciences, a CIRM training grant fellowship (No. TG2-01158), and a Glenn foundation grant. J.Q. was partially supported by an AFAR/Ellison Medical Foundation postdoctoral fellowship. We also thank other members of Belmonte Laboratory at Salk Institute for helpful discussions, and May Schwarz and Peter Schwarz for dedicated administrative support.

\section{ABBREVIATIONS}

AFP, alpha-fetoprotein; ALB, albumin; CNS, central nervous system; EB, embryoid body; FBS, fetal bovine serum; GFP, green fluorescent protein; hESC, human embryonic stem cells; HLC, hepatocyte-like cell; iPSC, induced pluripotent stem cell; MEF, mouse embryonic fibroblast; NSC, neural stem cell; PAS, Periodic Acid Schiff; qPCR, quantitative PCR; RT, room temperature; $\mathrm{TH}$, Tyrosine hydroxylase; WD, Wilson's disease

\section{REFERENCES}

Ala, A., Walker, A.P., Ashkan, K., Dooley, J.S., and Schilsky, M.L. (2007). Wilson's disease. Lancet 369, 397-408.

Barthel, H., Hermann, W., Kluge, R., Hesse, S., Collingridge, D.R., Wagner, A., and Sabri, O. (2003). Concordant pre- and postsynaptic deficits of dopaminergic neurotransmission in neurologic Wilson disease. AJNR Am J Neuroradiol 24, 234-238.

Chen, Y.F., Tseng, C.Y., Wang, H.W., Kuo, H.C., Yang, V.W., and Lee, O.K. (2012). Rapid generation of mature hepatocyte-like cells from human induced pluripotent stem cells by an efficient three-step protocol. Hepatology 55, 1193-1203.

Curtis, D., Durkie, M., Balac, P., Sheard, D., Goodeve, A., Peake, I., Quarrell, O., and Tanner, S. (1999). A study of Wilson disease mutations in Britain. Hum Mutat 14, 304-311.

Forbes, J.R., and Cox, D.W. (2000). Copper-dependent trafficking of Wilson disease mutant ATP7B proteins. Hum Mol Genet 9, 1927-1935.

Huster, D., Kuhne, A., Bhattacharjee, A., Raines, L., Jantsch, V., Noe, J., Schirrmeister, W., Sommerer, I., Sabri, O., Berr, F., et al. (2012). Diverse functional properties of Wilson disease ATP7B variants. Gastroenterology 142, 947-956.

Huster, D., Weizenegger, M., Kress, S., Mossner, J., and Caca, K. (2004). Rapid detection of mutations in Wilson disease gene ATP7B by DNA strip technology. Clin Chem Lab Med 42, 507-510.

Kenney, S.M., and Cox, D.W. (2007). Sequence variation database for the Wilson disease copper transporter, ATP7B. Hum Mutat 28, 1171-1177.

Lepori, M.B., Lovicu, M., Dessi, V., Zappu, A., Incollu, S., Zancan, L.,
Giacchino, R., lorio, R., Vajro, P., Maggiore, G., et al. (2007). Twenty-four novel mutations in Wilson disease patients of predominantly Italian origin. Genet Test 11, 328-332.

Li, M., Suzuki, K., Qu, J., Saini, P., Dubova, I., Yi, F., Lee, J., Sancho-Martinez, I., Liu, G.H., and Izpisua Belmonte, J.C. (2011a). Efficient correction of hemoglobinopathy-causing mutations by homologous recombination in integration-free patient iPSCs. Cell Res 21, 1740-1744.

Li, W., Sun, W., Zhang, Y., Wei, W., Ambasudhan, R., Xia, P., Talantova, M., Lin, T., Kim, J., Wang, X., et al. (2011b). Rapid induction and long-term self-renewal of primitive neural precursors from human embryonic stem cells by small molecule inhibitors. Proc Natl Acad Sci U S A 108, 8299-8304.

Liu, G.H., Barkho, B.Z., Ruiz, S., Diep, D., Qu, J., Yang, S.L., Panopoulos, A.D., Suzuki, K., Kurian, L., Walsh, C., et al. (2011a). Recapitulation of premature ageing with iPSCs from Hutchinson-Gilford progeria syndrome. Nature 472, 221-225.

Liu, G.H., Suzuki, K., Qu, J., Sancho-Martinez, I., Yi, F., Li, M., Kumar, S., Nivet, E., Kim, J., Soligalla, R.D., et al. (2011b). Targeted gene correction of laminopathy-associated LMNA mutations in patient-specific iPSCs. Cell Stem Cell 8, 688-694.

Lorincz, M.T. (2010). Neurologic Wilson's disease. Ann N Y Acad Sci 1184, 173-187.

Murata, Y., Yamakawa, E., lizuka, T., Kodama, H., Abe, T., Seki, Y., and Kodama, M. (1995). Failure of copper incorporation into ceruloplasmin in the Golgi apparatus of LEC rat hepatocytes. Biochem Biophys Res Commun 209, 349-355.

Saito, T., Okabe, M., Hosokawa, T., Kurasaki, M., Hata, A., Endo, F., Nagano, K., Matsuda, I., Urakami, K., and Saito, K. (1999). Immunohistochemical determination of the Wilson Copper-transporting P-type ATPase in the brain tissues of the rat. Neurosci Lett 266, 13-16.

Schilsky, M.L. (2009). Wilson disease: current status and the future. Biochimie 91, 1278-1281.

Song, Z., Cai, J., Liu, Y., Zhao, D., Yong, J., Duo, S., Song, X., Guo, Y., Zhao, Y., Qin, H., et al. (2009). Efficient generation of hepatocyte-like cells from human induced pluripotent stem cells. Cell Res 19, 1233-1242.

Stapelbroek, J.M., Bollen, C.W., van Amstel, J.K., van Erpecum, K.J., van Hattum, J., van den Berg, L.H., Klomp, L.W., and Houwen, R.H. (2004). The H1069Q mutation in ATP7B is associated with late and neurologic presentation in Wilson disease: results of a meta-analysis. J Hepatol 41, 758-763.

Strecker, K., Schneider, J.P., Barthel, H., Hermann, W., Wegner, F., Wagner, A., Schwarz, J., Sabri, O., and Zimmer, C. (2006). Profound midbrain atrophy in patients with Wilson's disease and neurological symptoms? J Neurol 253, 1024-1029.

Takahashi, K., Tanabe, K., Ohnuki, M., Narita, M., Ichisaka, T., Tomoda, K., and Yamanaka, S. (2007). Induction of pluripotent stem cells from adult human fibroblasts by defined factors. Cell 131, 861-872.

Terada, K., Aiba, N., Yang, X.L., lida, M., Nakai, M., Miura, N., and Sugiyama, T. (1999). Biliary excretion of copper in LEC rat after introduction of copper transporting P-type ATPase, ATP7B. FEBS Lett 448, 53-56.

Walshe, J.M. (1962). Wilson's disease. The presenting symptoms. Arch Dis Child 37, 253-256. 
Wilson, S. (1912). Progressive lenticular degeneration: A familiar nervous disease associated with cirhosis of the liver. Brain 34, 295-509.

Yi, F., Liu, G.H., and Izpisua Belmonte, J.C. (2012). Human induced pluripotent stem cells derived hepatocytes: rising promise for disease modeling, drug development and cell therapy. Protein
Cell 3, 246-250.

Zhang, S., Chen, S., Li, W., Guo, X., Zhao, P., Xu, J., Chen, Y., Pan, Q., Liu, X., Zychlinski, D., et al. (2011). Rescue of ATP7B function in hepatocyte-like cells from Wilson's disease induced pluripotent stem cells using gene therapy or the chaperone drug curcumin. Hum Mol Genet 20, 3176-3187. 